\title{
Modular functions and the uniform distribution of CM points
}

W. Duke

\section{Introduction} by

The classical $j$-function is defined for $z$ in the upper half plane $\mathcal{H}$

$$
j(z)=\frac{\left(1+240 \sum_{n=1}^{\infty} \sum_{m \mid n} m^{3} q^{n}\right)^{3}}{q \prod_{n=1}^{\infty}\left(1-q^{n}\right)^{24}}=q^{-1}+744+196884 q+\cdots,
$$

where $q=e(z)=e^{2 \pi i z}$. For $-d$ a negative fundamental discriminant, meaning that it is the discriminant of an imaginary quadratic field $K$, let

$$
z_{d}= \begin{cases}\frac{i \sqrt{d}}{2} & \text { if } d \equiv 0(\bmod 4), \\ \frac{-1+i \sqrt{d}}{2} & \text { if } d \equiv 3(\bmod 4) .\end{cases}
$$

The $j$-function has the remarkable property that $j\left(z_{d}\right)$ is an algebraic integer of degree $h(-d)$, the class number of $K=\mathbb{Q}\left(z_{d}\right)$. In fact, $K\left(j\left(z_{d}\right)\right)$ is the Hilbert class field of $K$ [3]. The first few values of $j\left(z_{d}\right)$ are:

$$
\begin{gathered}
j\left(z_{3}\right)=0, \quad j\left(z_{4}\right)=12^{3}, \quad j\left(z_{7}\right)=-15^{3}, \quad j\left(z_{8}\right)=20^{3}, \quad j\left(z_{11}\right)=-32^{3} \\
j\left(z_{15}\right)=\frac{-191025-85995 \sqrt{5}}{2}, \quad j\left(z_{19}\right)=-96^{3}, \quad j\left(z_{20}\right)=632000+282880 \sqrt{5},
\end{gathered}
$$

and $j\left(z_{23}\right)$ is the real root of $x^{3}+3491750 x^{2}-5151296875 x+23375^{3}=0$.

In general, the sum of the conjugates of $j\left(z_{d}\right)$, which we will denote by $\operatorname{Tr} j\left(z_{d}\right)$ for $d>4$, is an ordinary integer that defines an interesting arithmetic function of $d$. Thus, for the non-integral values listed above:

$\operatorname{Tr} j\left(z_{15}\right)=-191025, \quad \operatorname{Tr} j\left(z_{20}\right)=1264000, \quad \operatorname{Tr} j\left(z_{23}\right)=-3491750$.

2000 Mathematics Subject Classification: 11F37

Research supported in part by NSF Grant DMS-0355564. 
Concerning its size for large $d$, it is easy to show that for any $\alpha>1 / 2$

$$
\operatorname{Tr} j\left(z_{d}\right)=(-1)^{d} e^{\pi \sqrt{d}}+\mathrm{O}\left(e^{\alpha \pi \sqrt{d}}\right) .
$$

We will provide a much more precise asymptotic result. To state this, define for any $c \in \mathbb{Z}^{+}$the exponential sum

$$
S_{d}(c)=\sum_{x^{2} \equiv-d(c)} e(2 x / c) .
$$

As a special case of Theorem 1 proven below, we will show that

$$
\frac{1}{h(-d)}\left(\operatorname{Tr} j\left(z_{d}\right)-\sum_{\substack{0<c<2 \sqrt{d} \\ c=0(4)}} \frac{1}{2} S_{d}(c) e^{4 \pi \sqrt{d} / c}\right) \rightarrow 720
$$

as $d \rightarrow \infty$, with $-d$ a fundamental discriminant. That an asymptotic result of this type should hold was recently predicted by Bruinier, Jenkins and Ono [2] (see $\S 2$ below).

To see the significance of the constant 720 , consider the polynomial algebra $\mathbb{C}[j]$ of all "nearly holomorphic" modular functions for $\Gamma=\operatorname{PSL}(2, \mathbb{Z})$. The Laurent expansion $f(q)=\sum a_{n} q^{n}$ of any $f \in \mathbb{C}[j]$ is completely determined by its principal part

$$
f^{-}(z)=\sum_{n<0} a_{n} q^{n}
$$

Atkin (see [10]) introduced a canonical functional $\varphi$ on $\mathbb{C}[j]$ defined by

$$
\varphi(f)=a_{0}-24 \sum_{n<0} a_{n} \sigma(-n)
$$

where $\sigma(n)$ is the sum of the divisors of $n$. This has an equivalent formulation in terms of the regularized integral of $f$ [1, Thm 9.2]:

$$
\varphi(f)=\lim _{Y \rightarrow \infty} \frac{3}{\pi} \iint_{\mathcal{F}_{Y}} f(z) y^{-2} d x d y
$$

where $\mathcal{F}_{Y}=\{z \in \mathcal{F}: \operatorname{Im} z \leq Y\}$ is a truncation of the usual fundamental domain for $\Gamma$ :

$\mathcal{F}=\left\{z \in \mathcal{H}:-\frac{1}{2} \leq \operatorname{Re} z \leq 0\right.$ and $|z| \geq 1$ or $0<\operatorname{Re} z<\frac{1}{2}$ and $\left.|z|>1\right\}$. In particular, the "average" value of $j$ is 720 since from (4)

$$
\varphi(j)=720 \text {. }
$$

To give the generalization of (3), recall that a positive definite integral binary quadratic form

$$
Q(x, y)=a x^{2}+b x y+c y^{2}
$$


with discriminant $-d=b^{2}-4 a c$ is said to be reduced if the associated root of $Q(x, 1)=0$ in $\mathcal{H}$, called a CM point and given by

$$
z_{Q}=\frac{-b+i \sqrt{d}}{2 a}
$$

is in $\mathcal{F}$. For any negative $-d \equiv 0,1(\bmod 4)($ a negative discriminant) there is a principal form of discriminant $-d$ given by $x^{2}+\frac{d}{4} y^{2}$ if $d$ is even and by $x^{2}+x y+\frac{d+1}{4} y^{2}$ otherwise, that is reduced and whose corresponding CM point is $z_{d}$ of (1). We will always assume that $z_{Q}$ corresponds to a reduced form of discriminant $-d$. For any function $f$ on $\mathcal{H}$ and any negative discriminant $-d$ define

$$
\operatorname{Tr} f\left(z_{d}\right)=\sum_{z_{Q}} f\left(z_{Q}\right)
$$

where any summand of the form $f\left(z_{3}\right)$ or $f\left(z_{4}\right)$ is multiplied by $1 / 3$ and $1 / 2$, respectively. Let $H(d)=\operatorname{Tr} 1\left(z_{d}\right)$, called the Hurwitz class number.

For $d>4$ with $-d$ a fundamental discriminant we have that $H(d)=h(-d)$, and it follows from the classical theory of complex multiplication that $\operatorname{Tr} j\left(z_{d}\right)$ coincides with our previous definition since the conjugates of $j\left(z_{d}\right)$ are precisely the values $j\left(z_{Q}\right)$, called singular moduli. The main result of this paper is the following asymptotic formula for $\operatorname{Tr} f\left(z_{d}\right)$ for large $d$ and any $f \in \mathbb{C}[j]$.

THEOREM 1. Suppose that $f \in \mathbb{C}[j]$. Then

$$
\frac{1}{h(-d)}\left(\operatorname{Tr} f\left(z_{d}\right)-\sum_{\operatorname{Im} z_{Q}>1} f^{-}\left(z_{Q}\right)\right) \rightarrow \varphi(f),
$$

as $d \rightarrow \infty$ with $-d$ a fundamental discriminant.

Behind this result lies the uniform distribution of CM points in $\mathcal{H}$ proven in 1988 [5]. We remark that the restriction to fundamental discriminants is unnecessary and can be relaxed to allow all negative discriminants. In fact, the proof becomes much easier if $d$ has a large square factor.

In the special case $f=j$, Theorem 1 gives (3) upon using (6) and the easily established identity:

$$
\sum_{\operatorname{Im} z_{Q} \geq 1} e\left(-z_{Q}\right)=\frac{1}{2} \sum_{\substack{0<c<2 \sqrt{d} \\ c \equiv 0(4)}} S_{d}(c) e^{4 \pi \sqrt{d} / c} .
$$

There is another corollary worth pointing out. As Atkin observed, setting $\langle f, g\rangle=\varphi(f g)$ defines an inner product on $\mathbb{Q}[j]$, which has a 
unique orthogonal basis

$$
A_{0}(j)=1, \quad A_{1}(j)=j-720, \quad A_{2}(j)=j^{2}-1640 j+269280, \ldots,
$$

with monic polynomials $A_{n} \in \mathbb{Q}[x]$ of degree $n$ [10]. It is clear that $\operatorname{Tr} A_{n}\left(j\left(z_{d}\right)\right)$ is a rational number. It follows from Theorem 1 that for any fixed $n \geq 1$

$$
\frac{1}{h(-d)}\left(\operatorname{Tr} A_{n}\left(j\left(z_{d}\right)\right)-\sum_{\operatorname{Im} z_{Q}>1} A_{n}^{-}\left(j\left(z_{Q}\right)\right)\right) \rightarrow 0,
$$

as $d \rightarrow \infty$ with $-d$ a fundamental discriminant. An interesting property of $A_{n}$ is that for any prime $p$, if $n_{p}$ is the number of (isomorphism classes of) supersingular elliptic curves over $\overline{\mathbb{F}}_{p}$, then $A_{n_{p}}$ has $p$-integral coefficients and its roots in $\overline{\mathbb{F}}_{p}$ are exactly the supersingular $j$-invariants (see [10]).

\section{Meromorphic modular forms of weight $3 / 2$}

Before turning to the proof of Theorem 1, I will briefly explain its connection to the rich theory connecting singular moduli with meromorphic modular forms of half-integral weight. This theory, initiated by Borcherds and Zagier, has stimulated much current research [14].

Consider, for example, the weight $3 / 2$ modular form

(7) $g(z)=\theta_{1}(z) \frac{E_{4}(4 z)}{\eta(4 z)^{6}}=\sum_{d \geq-1} B(d) q^{d}=q^{-1}-2+248 q^{3}-492 q^{4}+\cdots$,

defined in terms of the usual modular forms:

$\theta_{1}(z)=\sum_{n \in \mathbb{Z}}(-1)^{n} q^{n^{2}}, \quad E_{4}(z)=1+240 \sum_{n \geq 1} \frac{n^{3} q^{n}}{1-q^{n}}, \quad \eta(z)=q^{\frac{1}{24}} \prod_{n \geq 1}\left(1-q^{n}\right)$.

It is clear from (7) that the Fourier coefficient $B(d)$ is zero unless $d \equiv 0,3(\bmod 4)$. Zagier $[16]$ proved the following striking connection between the traces of singular moduli and the coefficients $B(d)$.

THEOREM 2 (Zagier). For any positive $d \equiv 0,3(\bmod 4)$

$$
B(d)=-\operatorname{Tr} j_{1}\left(z_{d}\right),
$$

where $j_{1}=j-744$.

Motivated by this result and its generalizations, Bruinier, Jenkins and Ono [2] have recently obtained explicit formulas for the Fourier coefficients of certain modular forms of half-integral weight for $\Gamma_{0}(4)$ 
like $g(z)$, that are holomorphic on $\mathcal{H}$ but can have poles in the cusps. For $g(z)$ their formula can be written as:

$$
B(d)=24 H(d)-(1+i) \sum_{\substack{c>0 \\ c \equiv 0(4)}} \frac{\delta(c)}{\sqrt{c}} K(d,-1 ; c) \sinh \left(\frac{4 \pi \sqrt{d}}{c}\right),
$$

where $\delta(c)=2$ unless $c \equiv 0(\bmod 8)$, when it is 1 , and

$$
K(m, n ; c)=\sum_{a(c)}\left(\frac{c}{a}\right) \bar{\varepsilon}_{a} e\left(\frac{m a+n \bar{a}}{c}\right) \quad \text { where } \quad \varepsilon_{a}= \begin{cases}1, & \text { if } a \equiv 1(4) ; \\ i, & \text { if } a \equiv 3(4)\end{cases}
$$

is a half-integral weight Kloosterman sum. As they mention, (9) is analogous to the formula of Rademacher for the partition function, but they derive it using the method of Poincare series. Based on it they observe that an asymptotic formula like (3) should hold, and that a proof would follow from strong enough estimates for certain sums of half-integral weight Kloosterman sums.

In this paper we realize this observation in a somewhat different form. Instead of using Zagier's identity, the proof of (3) and Theorem 1 given here is based directly on methods used in [5] to establish the uniform distribution of CM points. One ingredient is the identification of the sum of a weight zero Maass form over CM points, called a hyperbolic Weyl sum, with a Fourier coefficient of a weight $1 / 2$ Maass form. This predecessor of Zagier's identity in the non-holomorphic cusp form case goes back to Maass [12] and was put in a more explicit form by Katok and Sarnak [11]. The estimation of the Fourier coefficient, which is at the heart of the proof of Theorem 1, does rely ultimately on non-trivial bounds for sums of half-integral weight Kloosterman sums proven by Iwaniec [8]. For our purposes here we need only adapt this known method to a modular function with exponential growth in the cusp, regularizing with a certain Poincaré series. While $\operatorname{Tr} f\left(z_{d}\right)$ is, after [16], the Fourier coefficient of an appropriate weight $3 / 2$ modular form for any $f \in \mathbb{C}[j]$ (provided $a_{0}=0$ ), the method used here does not apply to modular forms whose Fourier coefficients are not of this form.

As a by-product of our analysis we will obtain a "Kloosterman sum" proof of Zagier's identity (8). This is done by combining the formula (9) with a similar one for $\operatorname{Tr} j_{1}\left(z_{d}\right)$, which is easily obtained directly. The following formula valid for any discriminant $-d$ will be proven in $\S 4$ :

$$
\operatorname{Tr} j_{1}\left(z_{d}\right)=-24 H(d)+\sum_{c \equiv 0(4)} S_{d}(c) \sinh \left(\frac{4 \pi \sqrt{d}}{c}\right),
$$


where $S_{d}(c)$ is defined in (2). The fact that the Kloosterman sum in (10) can be evaluated is well known, in special cases, going back to Salié. Indeed, such evaluations are crucial in Iwaniec's estimates [8]. For general $c \equiv 0(\bmod 4)$ and any discriminant $-d$, however, the needed identity is apparently not well known. In $\S 5$ we prove that for all such $c, d$

$$
\delta(c)(1+i) K(d,-1 ; c)=\sqrt{c} S_{d}(c) .
$$

Thus (9) and (11) combine to give Theorem 2.

We remark that it should be possible to employ variations on this technique to prove more of Zagier's identities from [16]. This is clear for those obtained from (8) by Hecke operators but the formulas in [2] are not limited to these cases.

\section{Uniform distribution of $\mathrm{CM}$ points}

In this section we will prove Theorem 1 . For any nonnegative integer $m$ denote by $j_{m}$ the unique modular function such that $j_{m}(z)-$ $q^{-m}$ has a zero at $i \infty$. Thus $j_{0}=1, j_{1}=j-744$ and generally $j_{m} \in \mathbb{Z}[j]$. Since for any $f \in \mathbb{C}[j]$

$$
f(z)=\sum_{n \leq 0} a_{n} j_{-n}(z)
$$

it is clearly sufficient to prove Theorem 1 when $f=j_{m}$, for each $m \geq$ 1. In view of (5), we must only establish the following consequence of the uniform distribution of CM points.

Proposition 3. Fix any $Y \geq 1$ and $m \in \mathbb{Z}^{+}$. Then

$$
\frac{1}{h(-d)}\left(\sum_{z_{Q}} j_{m}\left(z_{Q}\right)-\sum_{\operatorname{Im} z_{Q}>Y} e\left(-m z_{Q}\right)\right) \rightarrow \frac{3}{\pi} \iint_{\mathcal{F}_{1}} j_{m}(z) y^{-2} d x d y
$$

as $d \rightarrow \infty$ with $-d$ a fundamental discriminant.

PROOF. Let $\psi: \mathbb{R}^{+} \rightarrow \mathbb{R}$ be a $C^{\infty}$ function with $\psi(y)=0$ for $y \leq Y$ and $\psi(y)=1$ for $y>Y+\varepsilon$, where $\varepsilon>0$ is fixed. Consider the Poincaré series for $m \in \mathbb{Z}$ :

$$
h_{m}(z)=\sum_{\gamma \in \Gamma_{\infty} \backslash \Gamma} e(-m \gamma z) \psi(\operatorname{Im} \gamma z)
$$

where $\Gamma_{\infty} \subset \Gamma$ is the subgroup of translations. Clearly we have that

$$
h_{m}(z)=e(-m z) \text { for } \operatorname{Im} z>Y+\epsilon .
$$

It follows that for a fixed $m \geq 0$ the function

$$
F(z)=F_{m}(z)=j_{m}(z)-h_{m}(z)
$$


is in $L^{2}(\Gamma \backslash H, d \mu)$, where $d \mu=y^{-2} d x d y$.

The function $F(z)$ thus has a spectral expansion convergent in norm (see [9])

$$
F(z)=c_{0}+\sum_{n>0} c_{n} u_{n}(z)+\frac{1}{4 \pi} \int_{-\infty}^{\infty} c(t) E\left(z, \frac{1}{2}+i t\right) d t .
$$

Here $\left\{u_{n}\right\}_{n \geq 1}$ is an orthonormal basis of Maass cusp forms and $E(z, s)$ is the Eisenstein series, given for $\operatorname{Re} s>1$ by

$$
E(z, s)=\sum_{\gamma \in \Gamma_{\infty} \backslash \Gamma}(\operatorname{Im} \gamma z)^{s} .
$$

Thus $\triangle u_{n}=\lambda_{n} u_{n}$ where the eigenvalues $\lambda_{n}$ of the hyperbolic Laplacian $\triangle=-y^{2}\left(\partial_{x}^{2}+\partial_{y}^{2}\right)$ are ordered so that $0<\lambda_{1} \leq \lambda_{2} \leq \cdots$. It is easily checked that $\iint_{\mathcal{F}} d \mu=\pi / 3$ and for $m>0$

$$
c_{0}=\frac{3}{\pi} \iint_{\mathcal{F}} F_{m}(z) d \mu=\frac{3}{\pi} \iint_{\mathcal{F}_{1}} j_{m}(z) y^{-2} d x d y .
$$

Since $F(z)$ is infinitely differentiable and of rapid decay at $\infty$, it is known [9] that (14) converges uniformly of compact subsets of $H$ and in fact a standard refinement of the proof given in [9] yields the bounds

$$
c_{n} \ll n^{-A} \text { and } c(t) \ll\left(\frac{1}{4}+t^{2}\right)^{-A},
$$

for any $A>0$, the implied constants depending only on $\psi, m$ and $A$.

By (14) we have

$$
\begin{aligned}
& \sum_{z_{Q}} j_{m}\left(z_{Q}\right)-\sum_{z_{Q}} e\left(-m z_{Q}\right) \psi\left(\operatorname{Im} z_{Q}\right)= \\
& h(-d) c_{0}+\sum_{n>0} c_{n} \sum_{z_{Q}} u_{n}\left(z_{Q}\right)+\frac{1}{4 \pi} \int_{-\infty}^{\infty} c(t) \sum_{z_{Q}} E\left(z_{Q}, \frac{1}{2}+i t\right) d t .
\end{aligned}
$$

We quote from $[6,(12.1)$ p. 35] the estimate for the hyperbolic "Weyl sum"

$$
\sum_{z_{Q}} u_{n}\left(z_{Q}\right) \ll n^{6} d^{\frac{1}{2}-\frac{1}{28}+\epsilon},
$$

whose proof relies on the non-trivial bound for the Fourier coefficients of half-integral weight Maass forms proven in [5]. For the continuous spectrum contribution to (17) we have the Burgess subconvexity bound for the Dirichlet $L$-function

$$
L_{-d}(s)=\sum_{n \geq 1}\left(\frac{-d}{n}\right) n^{-s}
$$


on $\operatorname{Re} s=1 / 2$, which gives

$$
\sum_{z_{Q}} E\left(z_{Q}, s\right) \ll d^{\frac{1}{4}}\left|L_{-d}(s)\right| \ll|s| d^{\frac{1}{2}-\frac{1}{16}+\epsilon} .
$$

Combining (15-18) with Siegel's theorem

$$
h(-d) \gg_{\epsilon} d^{\frac{1}{2}-\epsilon}
$$

(see [4]), we get a smoothed version of (13):

$$
\frac{1}{h(-d)}\left(\sum_{z_{Q}} j_{m}\left(z_{Q}\right)-\sum_{z_{Q}} e\left(-z_{Q}\right) \psi\left(\operatorname{Im} z_{Q}\right)\right)=c_{0}+\mathrm{O}\left(d^{-\delta}\right)
$$

for any $\delta<1 / 28$, where $c_{0}=\frac{3}{\pi} \iint_{\mathcal{F}_{1}} j_{m}(z) y^{-2} d x d y$.

In order to un-smooth this result suppose $0<\varepsilon<1 / 4$ is fixed. Consider the incomplete Eisenstein series

$$
g(z)=\sum_{\gamma \in \Gamma_{\infty} \backslash \Gamma} \phi(\operatorname{Im} \gamma z)
$$

where $\phi: \mathbb{R}^{+} \rightarrow \mathbb{R}$ is a $C^{\infty}$ function supported in $(Y-\varepsilon, Y+2 \varepsilon)$ with $0 \leq \phi(y) \leq 1$ and $\phi(y)=1$ for $Y \leq y \leq Y+\varepsilon$. Now $g(z)$ has an expansion given by

$$
g(z)=\sqrt{\frac{3}{\pi}} \hat{\phi}(0)+\frac{1}{2 \pi i} \int_{\operatorname{Re} s=\frac{1}{2}} \hat{\phi}(t) E(z, s) d s,
$$

where

$$
\hat{\phi}(t)=\int_{0}^{\infty} \phi(y) y^{-s-1} d y
$$

satisfies $\hat{\phi}(s) \ll(|s|+1)^{-A}$ (see [9, sec. 7.3]). It follows that

$$
\#\left\{z_{Q}: Y \leq \operatorname{Im} z_{Q} \leq Y+\varepsilon\right\} \leq \sum_{z_{Q}} g\left(z_{Q}\right) \leq 4 \varepsilon h(-d)
$$

using (19) again, provided $d$ is sufficiently large. Finally, we get from (20) and (21) that for

$$
R(d)=\frac{1}{h(-d)}\left(\sum_{z_{Q}} j_{m}\left(z_{Q}\right)-\sum_{\operatorname{Im} z_{Q}>Y} e\left(-m z_{Q}\right)\right)
$$

we have $\lim \sup _{d} R(d) \leq c_{0}+4 e^{3 \pi} \varepsilon$ and $\liminf _{d} R(d) \geq c_{0}-4 e^{3 \pi} \varepsilon$. Now Proposition 3 follows since $\varepsilon$ is arbitrarily small. 


\section{An exact formula for $\operatorname{Tr} j_{m}\left(z_{d}\right)$}

We now prove (11) using a variation on the argument given above. It is no more difficult to consider $\operatorname{Tr} j_{m}\left(z_{d}\right)$ for any $m \in \mathbb{Z}^{+}$. Thus define

$$
S_{d}(m, c)=\sum_{x^{2} \equiv-d(c)} e(2 m x / c)
$$

so that $S_{d}(1, c)=S_{d}(c)$ from (2).

Proposition 4. For any $m \in \mathbb{Z}^{+}$and any discriminant $-d$ we have that

$$
\operatorname{Tr} j_{m}\left(z_{d}\right)=-24 H(d) \sigma(m)+\sum_{c \equiv 0(4)} S_{d}(m, c) \sinh \left(\frac{4 \pi m \sqrt{d}}{c}\right),
$$

the series being convergent.

PROOF. Define for $y>0$ the function $V_{s}(y)=2 \pi y^{1 / 2} I_{s-1 / 2}(2 \pi y)$, where $I_{s-1 / 2}$ is the usual Bessel function so that

$$
V_{1}(y)=2 \sinh (2 \pi y) .
$$

For $m \in \mathbb{Z}^{+}$consider the Poincaré series

$$
j_{m}(z, s)=-24 \sigma(m)+\sum_{\gamma \in \Gamma_{\infty} \backslash \Gamma} e(-m \operatorname{Re} \gamma z) V_{s}(m \operatorname{Im} \gamma z)
$$

which converges absolutely for $\operatorname{Re} s>1$. It is readily seen that $j_{m}(\gamma z, s)=j_{m}(z, s)$ for $\gamma \in \Gamma$ and $\triangle j_{m}(z, s)=s(1-s) j_{m}(z, s)$. Niebur [13] showed that $j_{m}(z, s)$ has an analytic continuation to $s=1$ and that

$$
j_{m}(z)=j_{m}(z, 1) \text {. }
$$

A standard calculation using the orbits of the $\mathrm{CM}$ points under $\Gamma_{\infty} \backslash \Gamma$ shows that for $\operatorname{Re} s>1$

$$
\operatorname{Tr} j_{m}\left(z_{d}, s\right)=-24 H(d) \sigma(m)+\frac{1}{2} \sum_{\substack{c>0 \\ c \equiv 0(4)}} S_{d}(m, c) V_{s}\left(2 m \frac{\sqrt{d}}{c}\right) .
$$

Using (25) and (23) we can finish the proof, since it follows easily from well known results (see e.g. [7]) that the series (26) converges uniformly for $s \in[1,2]$, say. 


\section{Kloosterman sums}

Finally, we prove the Kloosterman sums identity (12), which implies Theorem 2 and is of independent interest. Recall that from (10) and (2),

$$
K(m, n ; c)=\sum_{a(c)}\left(\frac{c}{a}\right) \bar{\varepsilon}_{a} e\left(\frac{m a+n \bar{a}}{c}\right) \quad \text { and } \quad S_{d}(c)=\sum_{x^{2} \equiv-d(c)} e(2 x / c) .
$$

PROPOSITION 5. For $c$ a positive integer with $c \equiv 0(\bmod 4)$ and $d$ any integer with $d \equiv 0,3(\bmod 4)$ we have

$$
\delta(c)(1+i) K(d,-1 ; c)=\sqrt{c} S_{d}(c) .
$$

where $\delta(c)=1$ if $8 \mid c$ and $\delta(c)=2$ otherwise.

PROOF. We shall adapt an elegant method found recently by Toth [15], whose proof applies directly when $8 \mid c$, where no restriction on $d$ is necessary. In general, we have for any $b, d$ and $c>0$

$$
c \sum_{x^{2} \equiv-d(c)} e(b x / c)=\sum_{x(c)} e(b x / c) \sum_{a(c)} e\left(\frac{a\left(x^{2}+d\right)}{c}\right)=\sum_{a(c)} G(a, b ; c) e(a d / c)
$$

in terms of the Gauss sum $G(a, b ; c)=\sum_{x(c)} e\left(\frac{a x^{2}+b x}{c}\right)$. It is well known that $G(a, b ; c)$ vanishes if $n=(a, c)>1$ unless $n \mid b$, in which case

$$
G(a, b ; c)=n G\left(\frac{a}{n}, \frac{b}{n} ; \frac{c}{n}\right) .
$$

Thus we have

$$
c S_{d}(c)=\sum_{n \mid c} \sum_{\substack{a(c) \\(a, c)=n}} G(a, 2 ; c) e(a d / c),
$$

and $G(a, 2 ; c)=0$ in the inner sum unless $n=1$ or $n=2$. If $n=2$ we have $G(a, 2 ; c)=2 G\left(\frac{a}{2}, 1 ; \frac{c}{2}\right)$, which vanishes when $8 \mid c$.

Proceeding under this assumption

$$
c S_{d}(c)=\sum_{(a, c)=1} G(a, 2 ; c) e(a d / c) .
$$

Since when $(a, c)=1$ we have $G(a, 2 ; c)=e(-\bar{a} / c) G(a, 0 ; c)$, we may write

$$
c S_{d}(c)=G(1,0 ; c) \sum_{(a, c)=1} \frac{G(a, 0 ; c)}{G(1,0 ; c)} e(a d / c) e(-\bar{a} / c) .
$$

Now for $c \equiv 0(4)$ we have $\frac{G(a, 0 ; c)}{G(1,0 ; c)}=\left(\frac{c}{a}\right) \bar{\varepsilon}_{a}$ and also it is well known that $G(1,0 ; c)=(1+i) \sqrt{c}$. Thus $(27)$ holds in case $8 \mid c$ for any $d$. 
Suppose now that $c=4 q$ where $q$ is odd. A simple application of the Chinese remainder theorem and quadratic reciprocity gives [8, Lemma 2]:

$$
K(m, n ; c)=\left(\cos \frac{\pi(m+n)}{2}-\sin \frac{\pi(m+n)}{2}\right)(1-i) \varepsilon_{q} S(\overline{4} m, \overline{4} n ; q),
$$

where $S(m, n ; q)=\sum_{a(q)}\left(\frac{a}{q}\right) e\left(\frac{m a+n \bar{a}}{q}\right)$ is a Salié sum.

Suppose first that $d=4 D$. Then

$$
K(d,-1 ; c)=(1-i) \varepsilon_{q} S(D,-\overline{4} ; q) .
$$

Since

$$
S_{d}(c)=2 \sum_{x^{2} \equiv-D(q)} e(x / q)
$$

by (28) we get as before that

$$
\frac{q}{2} S_{d}(c)=\sum_{(a, q)=1} G(a, 1 ; q) e(a D / q)=G(1,0 ; q) \sum_{(a, q)=1} \frac{G(a, 0 ; q)}{G(1,0 ; q)} e\left(\frac{D a-\overline{4} \bar{a}}{q}\right),
$$

but now we using that for $(a, q)=1$ we have $G(a, 1 ; q)=e(-\overline{4} \bar{a} / q) G(a, 0 ; q)$. Now $\frac{G(a, 0 ; q)}{G(1,0 ; q)}=\left(\frac{a}{q}\right)$ and also $G(1,0 ; q)=\varepsilon_{q} \sqrt{q}$, so we have

$$
\sqrt{c} S_{d}(c)=4 \varepsilon_{q} S(D,-\overline{4} ; q) .
$$

From this and (32) we derive (27). In case $d=4 D-1$ we start with

$$
S_{d}(c)=2 e\left(\frac{1}{2 q}\right) \sum_{x^{2}+x \equiv-D(q)} e(x / q)=-2 \sum_{x^{2} \equiv \overline{4}-D(q)} e(x / q),
$$

since $e\left(\frac{1}{2 q}\right) e\left(\frac{-\overline{2}}{q}\right)=-1$, as $(1-2 \overline{2})$ is odd. Now proceed as before to get

$$
\sqrt{c} S_{d}(c)=-4 \varepsilon_{q} S(D-\overline{4},-\overline{4} ; q) .
$$

Using again (31) we have

$$
K(d,-1 ; c)=(i-1) \varepsilon_{q} S(\overline{4}(4 D-1),-\overline{4} ; q)
$$

giving (27).

We remark that (27) does not hold in general if $d \equiv 1,2(4)$ and $8 \nmid c$, since $K(d,-1 ; c)$ need not vanish yet $S_{d}(c)$ does.

Acknowedgement: I thank Ken Ono for introducing me to the problems treated in this paper and for his comments on an earlier version. 


\section{References}

[1] Borcherds, Richard E. Automorphic forms with singularities on Grassmannians. Invent. Math. 132 (1998), no. 3, 491-562

[2] Brunier, Jan Hendrik; Jenkins, Paul and Ono, Ken. Hilbert class polynomials and traces of singular moduli, preprint.

[3] Cox, David A. Primes of the form $x^{2}+n y^{2}$. Fermat, class field theory and complex multiplication. A Wiley-Interscience Publication. John Wiley and Sons, Inc., New York, 1989.

[4] Davenport, Harold. Multiplicative number theory. Third edition. Revised and with a preface by Hugh L. Montgomery. Graduate Texts in Mathematics, 74. Springer-Verlag, New York, 2000.

[5] Duke, W. Hyperbolic distribution problems and half-integral weight Maass forms. Invent. Math. 92 (1988), no. 1, 73-90.

[6] Duke, W.; Friedlander, J.; Iwaniec, H. Class group L-functions. Duke Math. J. 79 (1995), no. 1, 1-56.

[7] Duke, W.; Friedlander, J. B.; Iwaniec, H. Equidistribution of roots of a quadratic congruence to prime moduli. Ann. of Math. (2) 141 (1995), no. 2, 423-441.

[8] Iwaniec, Henryk. Fourier coefficients of modular forms of half-integral weight. Invent. Math. 87 (1987), no. 2, 385-401.

[9] Iwaniec, Henryk. Spectral methods of automorphic forms. Second edition. Graduate Studies in Mathematics, 53. American Mathematical Society, Providence, RI; Revista Matemtica Iberoamericana, Madrid, 2002.

[10] Kaneko, M.; Zagier, D. Supersingular $j$-invariants, hypergeometric series, and Atkin's orthogonal polynomials. Computational perspectives on number theory (Chicago, IL, 1995), 97-126, AMS/IP Stud. Adv. Math., 7, Amer. Math. Soc., Providence, RI, 1998.

[11] Katok, Svetlana; Sarnak, Peter. Heegner points, cycles and Maass forms. Israel J. Math. 84 (1993), no. 1-2, 193-227.

[12] Maass, Hans. Über die räumliche Verteilung der Punkte in Gittern mit indefiniter Metrik. Math. Ann. 1381959 287-315.

[13] Niebur, Douglas. A class of nonanalytic automorphic functions. Nagoya Math. J. 52 (1973), 133-145.

[14] Ono, Ken. The web of modularity: arithmetic of the coefficients of modular forms and $q$-series. CBMS Regional Conference Series in Mathematics, 102. Published for the Conference Board of the Mathematical Sciences, Washington, DC; by the American Mathematical Society, Providence, RI, 2004.

[15] Tóth, Á ,. On the evaluation of Salié sums, to appear in Proc. AMS.

[16] Zagier, Don. Traces of singular moduli. Motives, polylogarithms and Hodge theory, Part I (Irvine, CA, 1998), 211-244, Int. Press Lect. Ser., 3, I, Int. Press, Somerville, MA, 2002.

\section{UCLA Mathematics Department Box 951555, Los Angeles, CA 90095-1555}

Email: duke@math.ucla.edu 